\title{
OPTIMAL STOPPING WHEN THE ABSORBING BOUNDARY IS FOLLOWING AFTER
}

\author{
MASAHIKO EGAMI AND TADAO ORYU
}

\begin{abstract}
We consider a new type of optimal stopping problems where the absorbing boundary moves as the state process $X$ attains new maxima $S$. More specifically, we set the absorbing boundary as $S-b$ where $b$ is a certain constant. This problem is naturally connected with excursions from zero of the reflected process $S-X$. We examine this constrained optimization with the state variable $X$ as a spectrally negative Lévy process. The problem is in nature a two-dimensional one. The threshold strategy given by the path of just $X$ is not in fact optimal. It turns out, however, that we can reduce the original problem to an infinite number of one-dimensional optimal stopping problems, and we find explicit solutions.

This work is motivated by the bank's profit maximization with the constraint that it maintain a certain level of leverage ratio. When the bank's asset value severely deteriorates, the bank's required capital requirement shall be violated. This situation corresponds to $X<S-b$ in our setting. This model may well describe a real-life situation where even a large bank can fail because the absorbing boundary is keeping up with the size of the bank.
\end{abstract}

Key words: Optimal stopping; excursion theory; spectrally negative Lévy processes; scale functions. Mathematics Subject Classification (2010) : Primary: 60G40 Secondary: 60J75

\section{INTRODUCTION}

The literature about optimal stopping problems and their applications is immense. In an infinite horizon problem, with one-dimensional continuous diffusions as the state variable, a full characterization of the value function and of optimal stopping rule is known and the methodology for solution has been established. See, for example, Dynkin [13], Alvarez [2], Dayanik and Karatzas [11]. For spectrally negative Lévy processes, or Lévy processes with only negative jumps, a number of authors have succeeded in extending the classical results by using the scale functions. We just name a few here : [5, 6] for stochastic games, [4, 19, 21] for the optimal dividend problem, [1, 3] for American and Russian options, and [16, 20] for credit risk. However, the solution techniques presented in each paper are more or less problem-specific and no characterization of the value function is yet known. If the problem involves two state variables, then even for continuous diffusions, very few things are known in the literature.

We study a new type of optimal stopping problems. We let $X=\left(X_{t}, t \geq 0\right)$ be a spectrally negative Lévy process and denote by $Y$ the reflected process

$$
Y_{t}=S_{t}-X_{t}
$$

First draft: September 3, 2012 ; this version: August 18, 2014.

TThis work is in part supported by Grant-in-Aid for Scientific Research (B) No. 23330104 and No. 26285069, Japan Society for the Promotion of Science. We thank the participants for their valuable comments in the workshop "Topics in Lévy and jump models" at Osaka University. 
where $S_{t}=\sup _{u \in[0, t]} X_{u} \vee s$. We then consider an optimal stopping problem for both $X$ and $S$ in which the absorbing boundary is defined by $\left(S_{t}-b, t \geq 0\right)$ with $b$ as a positive constant. This means that while $X$ grows and keeps attaining new maxima, the absorbing boundary is accompanying with $S$. Hence an excursion from $S$, if greater than $b$, would bring $X$ to ruin. This situation is seen in the real world; for example, several large financial institutions failed in the last crisis in 2007-2008. One of the reasons is that, while becoming large banks, they maintain high leverage and accordingly, the banks are not so far way from the bankruptcy threshold. Instead, the bankruptcy threshold keeps up with the size of the banks. That is, despite the size of the bank, the risk of bankruptcy is not so much mitigated. This paper is motivated by this phenomenon. See Section 4 for details. While we take the example of banking, one can come up with other applications of this type, as long as the absorbing boundary is determined in relation to the state process' running maxima. For instance, a gambler may have a policy that he stops betting when his wealth $X$ goes below a certain level $b$ from that day's running maxima $S$.

An excursion theory for spectrally negative Lévy processes has been developed recently. See Bertoin [7] as a general reference. More specifically, an exit problem of the reflected process $Y$ was studied by Avram et al. [3], Pistorius [25] [26] and Doney [12].

In the above cited papers on optimal stopping problems, the optimal strategy is usually obtained by so-called "threshold strategy". That is, the player should stop and receive rewards on the first occasion when the state process enters a stopping region. In Lévy and other jump models, the authors first find the optimal threshold level and then prove its optimality by verifying the 'quasi-variational inequalities'. See Øksendal and Sulem [22]. Since the problem at hand involves two dimensions; one is $X$ and the other is $S$, finding and proving the overall optimal strategy may be challenging (as mentioned, no characterization in two-dimensional problems has been found). Recent developments on two-dimensional optimal stopping problems (involving $S$ and $X$ ) include Ott [23] and Guo and Zervos [17]. In the former paper, the author solves problems including a capped version of the Shepp-Shiryaev problem [27], and the latter paper is another contribution that extends [27].

In our particular situation, by looking at excursions that occur at each level of $S$, we reduce the problem to an infinite number of one-dimensional optimal stopping problems. We shall then find an explicit form of the solution, thanks to the results by Pistorius [26]. It turns out that the optimal stopping region can be shown in a diagram created by various values of $S$ and $S-X$ (e.g., Figure 6).

The rest of the paper is organized as follows. In Section 2, we formulate a mathematical model with a review of some important facts of spectrally negative Lévy processes, and then find an optimal threshold level in Section 3. We shall take the example of a bank's optimization in Section 4 and provide an explicit calculation.

\section{Mathematical Model}

Let the spectrally negative Levy process $X=\left\{X_{t} ; t \geq 0\right\}$ represent the state variable defined on the probability space $(\Omega, \mathcal{F}, \mathbb{P})$, where $\Omega$ is the set of all possible realization of the stochastic economy, and $\mathbb{P}$ is a probability measure defined on $\mathcal{F}$. We denote by $\mathbb{F}=\left\{\mathcal{F}_{t}\right\}_{t \geq 0}$ the filtration with respect to which $X$ is adapted and with the usual conditions being satisfied. The Laplace exponent $\psi$ of $X$ is given by

$$
\psi(\lambda)=\mu \lambda+\frac{1}{2} \sigma^{2} \lambda^{2}+\int_{(-\infty, 0)}\left(e^{\lambda x}-1-\lambda x \mathbb{1}_{(x>-1)}\right) \Pi(\mathrm{d} x),
$$


where $\mu \geq 0, \sigma \geq 0$, and $\Pi$ is a measure concentrated on $\mathbb{R} \backslash\{0\}$ satisfying $\int_{\mathbb{R}}\left(1 \wedge x^{2}\right) \Pi(\mathrm{d} x)<\infty$. It is well-known that $\psi$ is zero at the origin, convex on $\mathbb{R}_{+}$and has a right-continuous inverse:

$$
\Phi(q):=\sup \{\lambda \geq 0: \psi(\lambda)=q\}, \quad q \geq 0 .
$$

The running maximum process $S=\left\{S_{t} ; t \geq 0\right\}$ is defined by $S_{t}=\sup _{u \in[0, t]} X_{u} \vee s$. In addition, we write $Y$ for the reflected process defined by $Y_{t}=S_{t}-X_{t}$, and let $\zeta$ be the stopping time defined by

$$
\zeta:=\inf \left\{t \geq 0: Y_{t}>b\right\}, \quad b>0,
$$

the time of ruin. The payoff is composed of three parts; the running income to be received continuously until stopped or absorbed, the terminal reward part to be received when the process is stopped, and the penalty part incurred when the process is absorbed.

We consider the following optimal stopping problem and the value function $\bar{V}: \mathbb{R}^{2} \mapsto \mathbb{R}$ associated with initial values $X_{0}=x$ and $S_{0}=s$;

$$
\begin{aligned}
\bar{V}(x, s)= & \sup _{\tau \in \mathcal{S}} \mathbb{E}^{x, s}\left[\int_{0}^{\tau \wedge \zeta} e^{-q t} f\left(X_{t}, S_{t}\right) \mathrm{d} t\right. \\
& \left.+\mathbb{1}_{\{\tau<\zeta\}} e^{-q \tau} g\left(X_{\tau}, S_{\tau}\right)-\mathbb{1}_{\{\tau \geq \zeta\}} e^{-q \zeta} k\left(X_{\zeta}, S_{\zeta}\right)\right]
\end{aligned}
$$

where $\mathbb{P}^{x, s}(\cdot):=\mathbb{P}\left(\cdot \mid X_{0}=x, S_{0}=s\right)$ and $\mathbb{E}^{x, s}$ is the expectation operator corresponding to $\mathbb{P}^{x, s}, q \geq 0$ is the constant discount rate and $\mathcal{S}$ is the set of all $\mathbb{F}$-adapted stopping times. The running income function $f: \mathbb{R}^{2} \mapsto \mathbb{R}$ is a measurable function that satisfies

$$
\mathbb{E}^{x, s}\left[\int_{0}^{\infty} e^{-q t}\left|f\left(X_{t}, S_{t}\right)\right| \mathrm{d} t\right]<\infty .
$$

The reward function $g: \mathbb{R}^{2} \mapsto \mathbb{R}_{+}$and the penalty function $k: \mathbb{R}^{2} \mapsto \mathbb{R}_{+}$are assumed to be measurable. Our main purpose is to calculate $\bar{V}$ and to find the stopping time $\tau^{*}$ which attains the supremum.

For each Borel measurable function $l: \mathbb{R} \mapsto \mathbb{R}_{+}$, we define a stopping time $\tau(l)$ by

$$
\tau(l):=\inf \left\{t \geq 0: S_{t}-X_{t}>l\left(S_{t}\right)\right\}
$$

and define a set of stopping times $\mathcal{S}^{\prime}$ by

$$
\mathcal{S}^{\prime}:=\left\{\tau(l): l: \mathbb{R} \mapsto \mathbb{R}_{+}\right\}
$$

In other words, $\tau(l)$ is the first time the excursion $S-X$ from level, say $S=s$, becomes greater than some value $l(s)$.

When $l$ is constant, for example, $\bar{l} \equiv c$ on $\mathbb{R}$, we write

$$
\tau_{c}:=\inf \left\{t \geq 0: S_{t}-X_{t}>c\right\} .
$$

In particular, if $\bar{l} \equiv b$, then $\tau_{b}=\zeta$. Next we let $\mathcal{S}^{\prime}(b)$ be the set of stopping times defined by

$$
\mathcal{S}^{\prime}(b):=\{\tau(l): l(m) \leq b \text { for all } m \in \mathbb{R}\} .
$$

Note that if $\tau \in \mathcal{S}^{\prime}(b)$, then $\tau \leq \zeta$. The following lemma shows that it suffices to consider stopping times $\tau \in \mathcal{S}^{\prime}(b)$. 
Lemma 2.1. Let us define $u: \mathbb{R}^{2} \times \mathcal{S}^{\prime} \mapsto \mathbb{R}$ by

$$
\begin{aligned}
u(x, s ; \tau):= & \mathbb{E}^{x, s}\left[\int_{0}^{\tau \wedge \zeta} e^{-q t} f\left(X_{t}, S_{t}\right) \mathrm{d} t\right. \\
& \left.+\mathbb{I}_{\{\tau<\zeta\}} e^{-q \tau} g\left(X_{\tau}, S_{\tau}\right)-\mathbb{I}_{\{\tau \geq \zeta\}} e^{-q \zeta} k\left(X_{\zeta}, S_{\zeta}\right)\right]
\end{aligned}
$$

Then for any $\tau \in \mathcal{S}^{\prime}$, we can find $a \tau^{\prime} \in \mathcal{S}^{\prime}(b)$ such that $u(x, s ; \tau)=u\left(x, s ; \tau^{\prime}\right)$.

Proof. Set functions $l_{1}$ and $l_{2}$ by $l_{1}(s)>l_{2}(s)=b$ for some $s$ and $l_{1}(m)=l_{2}(m)$ on $m \neq s$. Then it is clear from the definition that $\tau\left(l_{1}\right)<\zeta$ if and only if $\tau\left(l_{2}\right)<\zeta$, and $\tau\left(l_{1}\right)=\tau\left(l_{2}\right)$ on $\left\{\tau\left(l_{1}\right)<\zeta\right\}$. Hence the right hand side of (2.1) for $\tau=\tau\left(l_{1}\right)$ and $\tau=\tau\left(l_{2}\right)$ are equal to each other. Hence the lemma is proved.

2.1. Note on the Optimal Strategy. We will reduce the original problem (2.1) to an infinite number of onedimensional optimal stopping problem and discuss the optimality of the proposed strategy (2.2). Let us denote by $\bar{f}: \mathbb{R}^{2} \mapsto \mathbb{R}$ the $q$-potential of $f$ where

$$
\bar{f}(x, s):=\mathbb{E}^{x, s}\left[\int_{0}^{\infty} e^{-q t} f\left(X_{t}, S_{t}\right) \mathrm{d} t\right] .
$$

From the strong Markov property of $(X, S)$, we have

$$
\begin{aligned}
& \mathbb{E}^{x, s}\left[\int_{0}^{\tau \wedge \zeta} e^{-q t} f\left(X_{t}, S_{t}\right) \mathrm{d} t\right] \\
= & \mathbb{E}^{x, s}\left[\int_{0}^{\infty} e^{-q t} f\left(X_{t}, S_{t}\right) \mathrm{d} t-\int_{\tau \wedge \zeta}^{\infty} e^{-q t} f\left(X_{t}, S_{t}\right) \mathrm{d} t\right] \\
= & \bar{f}(x, s)-\mathbb{E}^{x, s}\left[\mathbb{E}\left[\int_{\tau \wedge \zeta}^{\infty} e^{-q t} f\left(X_{t}, S_{t}\right) \mathrm{d} t \mid \mathcal{F}_{\tau \wedge \zeta}\right]\right] \\
= & \bar{f}(x, s)-\mathbb{E}^{x, s}\left[e^{-q(\tau \wedge \zeta)} \mathbb{E}^{X_{\tau \wedge \zeta}, S_{\tau \wedge \zeta}}\left[\int_{0}^{\infty} e^{-q t} f\left(X_{t}, S_{t}\right) \mathrm{d} t\right]\right] \\
= & \bar{f}(x, s)-\mathbb{E}^{x, s}\left[e^{-q(\tau \wedge \zeta)} \bar{f}\left(X_{\tau \wedge \zeta}, S_{\tau \wedge \zeta}\right)\right] \\
= & \bar{f}(x, s)-\mathbb{E}^{x, s}\left[\mathbb{1}_{\{\tau<\zeta\}} e^{-q \tau} \bar{f}\left(X_{\tau}, S_{\tau}\right)+\mathbb{1}_{\{\zeta \leq \tau\}} e^{-q \zeta} \bar{f}\left(X_{\zeta}, S_{\zeta}\right)\right] .
\end{aligned}
$$

Hence the value function $\bar{V}$ can be written as

$$
\bar{V}(x, s)=\bar{f}(x, s)+V(x, s),
$$

where

$$
V(x, s):=\sup _{\tau \in \mathcal{S}} \mathbb{E}^{x, s}\left[\mathbb{1}_{\{\tau<\zeta\}} e^{-q \tau}(g-\bar{f})\left(X_{\tau}, S_{\tau}\right)-\mathbb{1}_{\{\zeta \leq \tau\}} e^{-q \zeta}(k+\bar{f})\left(X_{\zeta}, S_{\zeta}\right)\right] .
$$

Since $\bar{f}(x, s)$ has nothing to do with the choice of $\tau$, we concentrate on $V(x, s)$.

By the dynamic programming principle, we can write $V(x, s)$ as

$$
\begin{aligned}
V(x, s) & =\sup _{\tau \in \mathcal{S}} \mathbb{E}^{x, s}\left[\mathbb{1}_{\{\tau<\theta\}} \mathbb{1}_{\{\tau<\zeta\}} e^{-q \tau}(g-\bar{f})\left(X_{\tau}, S_{\tau}\right)\right. \\
& \left.-\mathbb{1}_{\{\zeta<\theta\}} \mathbb{1}_{\{\zeta \leq \tau\}} e^{-q \zeta}(k+\bar{f})\left(X_{\zeta}, S_{\zeta}\right)+\mathbb{1}_{\{\theta<\tau \wedge \zeta\}} e^{-q \theta} V\left(X_{\theta}, S_{\theta}\right)\right],
\end{aligned}
$$


for any stopping time $\theta \in \mathcal{S}$. See, for example, Pham [24] page 97. Now we set $\theta=T_{s}$ in (2.5). For each level $S=s$ from which an excursion occurs, the value $S$ does not change during the excursion. Hence, during the first excursion interval from $S_{0}=s, \zeta=T_{s-b}^{-}$and $S_{t}=s$ for any $t \leq T_{s}$, and (2.5) can be written as the following one-dimensional problem for the state process $X$;

$$
\begin{aligned}
V(x, s)= & \sup _{\tau \in \mathcal{S}} \mathbb{E}^{x, s}\left[\mathbb{1}_{\left\{\tau<T_{s}\right\}} \mathbb{1}_{\left\{\tau<T_{s-b}^{-}\right\}} e^{-q \tau}(g-\bar{f})\left(X_{\tau}, s\right)\right. \\
& -\mathbb{1}_{\left\{T_{s-b}^{-}<T_{s}\right\}} \mathbb{1}_{\left\{T_{s-b}^{-} \leq \tau\right\}} e^{-q \tau}(k+\bar{f})\left(X_{T_{s-b}^{-}}, s\right) \\
& \left.+\mathbb{1}_{\left\{T_{s}<\tau \wedge T_{s-b}^{-}\right\}} e^{-q T_{s}} V(s, s)\right]
\end{aligned}
$$

Now we can look at only the process $X$ and find $\tau^{*} \in \mathcal{S}$.

In relation to (2.6), we consider the following one-dimensional optimal stopping problem as for $X$ and its value function $\widehat{V}: \mathbb{R}^{2} \mapsto \mathbb{R}$;

$$
\begin{aligned}
\widehat{V}(x, s) & =\sup _{\tau \in \mathcal{S}} \mathbb{E}^{x, s}\left[\mathbb{1}_{\left\{\tau<T_{s}\right\}} \mathbb{1}_{\left\{\tau<T_{s-b}^{-}\right\}} e^{-q \tau}(g-\bar{f})\left(X_{\tau}, s\right)\right. \\
& \left.-\mathbb{1}_{\left\{T_{s-b}^{-}<T_{s}\right\}} \mathbb{1}_{\left\{T_{s-b}^{-} \leq \tau\right\}} e^{-q \tau}(k+\bar{f})\left(X_{T_{s-b}^{-}}, s\right)+\mathbb{1}_{\left\{T_{s}<\tau \wedge T_{s-b}^{-}\right\}} e^{-q T_{s}} K\right],
\end{aligned}
$$

where $K \geq 0$ is a constant. Then the following lemma provides a sufficient condition for a threshold strategy to be optimal for (2.7). See also Theorem 2.2 in Øksendal and Sulem [22] for this type of verification theorem. Note that $V=\widehat{V}$ holds when $K=V(s, s)$, and $V(s, s)$ can be obtained by our solution method offered in Section 3 .

Lemma 2.2. Fix some $s \in \mathbb{R}$. If there exist $z^{*} \in(-\infty, s]$ and a function $w \in \mathcal{C}^{1}((-\infty, s]) \cap \mathcal{C}^{2}\left((-\infty, s) \backslash\left\{z^{*}\right\}\right)$ such that

(i) $w(s)=K$,

(ii) $(\mathcal{A}-q) w(x)=0$ and $w(x)>(g-\bar{f})(x, s)$ on $x \in\left(z^{*}, s\right)$,

(iii) $(\mathcal{A}-q) w(x)<0$ and $w(x)=(g-\bar{f})(x, s)$ on $x \in\left[s-b, z^{*}\right]$,

(iv) $w(x)=-(k+\bar{f})(x, s)$ on $x \in(-\infty, s-b)$,

then $w(x)=\widehat{V}(x, s)$ for every $x \in(-\infty, s]$ and the $\mathbb{F}$-stopping time $\tau^{*}=\inf \left\{t \geq 0 ; X_{t}<z^{*}\right\}$ gives supremum in (2.7). Note that

$$
\mathcal{A} w(x):=\mu w^{\prime}(x)+\frac{\sigma^{2}}{2} w^{\prime \prime}(x)+\int_{0}^{\infty} \Pi(\mathrm{d} y)\left[w(x+y)-w(x)-y w^{\prime}(x) \mathbb{l}_{\{-1<y\}}\right] .
$$


Proof. First we prove $w(x) \geq \widehat{V}(x, s)$ for every $x \in \mathbb{R}_{+}$. The Itô's rule (see e.g. Cont and Tankov[10] page 277) gives us

$$
\begin{aligned}
& e^{-q\left(t \wedge T_{s} \wedge T_{s-b}^{-}\right)} w\left(X_{t \wedge T_{s} \wedge T_{s-b}^{-}}\right) \\
= & w\left(X_{0}\right)-\int_{0}^{t \wedge T_{s} \wedge T_{s-b}^{-}} q e^{-q u} w\left(X_{u}\right) \mathrm{d} u \\
& +\mu \int_{0}^{t \wedge T_{s} \wedge T_{s-b}^{-}} e^{-q u} w^{\prime}\left(X_{u}\right) \mathrm{d} u+\sigma \int_{0}^{t \wedge T_{s} \wedge T_{s-b}^{-}} e^{-q u} w^{\prime}\left(X_{u}\right) \mathrm{d} B_{u} \\
& +\frac{\sigma^{2}}{2} \int_{0}^{t \wedge T_{s} \wedge T_{s-b}^{-}} e^{-q u} w^{\prime \prime}\left(X_{u}\right) \mathrm{d} u \\
& +\int_{0}^{t \wedge T_{s} \wedge T_{s-b}^{-}} \int_{0}^{\infty} \mathrm{d} u \Pi(\mathrm{d} y) e^{-q u}\left[w\left(X_{u}+y\right)-w\left(X_{s-}\right)-y w^{\prime}\left(X_{s-}\right) \mathbb{1}_{\{-1<y\}}\right] \\
& +\int_{0}^{t \wedge T_{s} \wedge T_{s-b}^{-}} \int_{0}^{\infty}(M(\mathrm{~d} u, \mathrm{~d} y)-\mathrm{d} u \Pi(\mathrm{d} y)) e^{-q u}\left[w\left(X_{u}+y\right)-w\left(X_{u-}\right)\right] \\
= & w\left(X_{0}\right)-\int_{0}^{t \wedge T_{s} \wedge T_{s-b}^{-}} q e^{-q u} w\left(X_{u}\right) \mathrm{d} u \\
& +\int_{0}^{t \wedge T_{s} \wedge T_{s-b}^{-}} e^{-q u} w^{\prime}\left(X_{u}\right) \mathrm{d} u+\sigma \int_{0}^{t \wedge T_{s} \wedge T_{s-b}^{-}} e^{-q u} w^{\prime}\left(X_{u}\right) \mathrm{d} B_{u} \\
& +\int_{0}^{t \wedge T_{s} \wedge T_{s-b}^{-}} e^{-q u}(\mathcal{A}-q) w\left(X_{u}\right) \mathrm{d} u \\
& +\int_{0}^{t \wedge T_{s} \wedge T_{s-b}^{-}} \int_{0}^{\infty}(M(\mathrm{~d} u, \mathrm{~d} y)-\mathrm{d} u \Pi(\mathrm{d} y)) e^{-q u}\left[w\left(X_{u}+y\right)-w\left(X_{u-}\right)\right]
\end{aligned}
$$

where we denote by $M$ the Poisson random measure associated with $X$. Since the process $\left\{X_{t \wedge T_{s} \wedge T_{s-b}^{-}}: t \geq 0\right\}$ does not leave the interval $[s-b, s]$, the integrals with respect to the Brownian motion $B$ and the compensated jump measure (i.e., the last term) are martingales. Therefore, by taking expectations, we have

$$
\begin{aligned}
w(x)= & \mathbb{E}^{x, s}\left[e^{-q\left(t \wedge T_{s} \wedge T_{s-b}^{-}\right)} w\left(X_{t \wedge T_{s} \wedge T_{s-b}^{-}}\right)\right] \\
& -\mathbb{E}^{x, s}\left[\int_{0}^{t \wedge T_{s} \wedge T_{s-b}^{-}} e^{-q u}(\mathcal{A}-q) w\left(X_{u}\right) \mathrm{d} u\right]
\end{aligned}
$$

By the assumption

$$
(\mathcal{A}-q) w(x) \leq 0, \quad x \in(-\infty, s]
$$

we have

$$
w(x) \geq \mathbb{E}^{x, s}\left[e^{-q\left(\tau \wedge T_{s} \wedge T_{s-b}^{-}\right)} w\left(X_{\tau \wedge T_{s} \wedge T_{s-b}^{-}}\right)\right], x \in(-\infty, s], \tau \in \mathcal{S} .
$$


Now, for any stopping time $\tau \in \mathcal{S}$, by using (i)-(iv),

$$
\begin{aligned}
& w(x) \geq \mathbb{E}^{x, s}\left[e^{-q\left(\tau \wedge T_{s} \wedge T_{s-b}^{-}\right)} w\left(X_{\tau \wedge T_{s} \wedge T_{s-b}^{-}}\right)\right] \\
& \geq \mathbb{E}^{x, s}\left[\mathbb{1}_{\left\{\tau<T_{s}\right\}} \mathbb{1}_{\left\{\tau<T_{s-b}^{-}\right\}} e^{-q \tau}(g-\bar{f})\left(X_{\tau}, s\right)\right.
\end{aligned}
$$

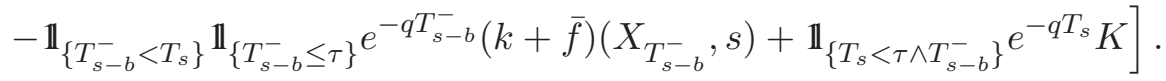

Taking the supremum over the set $\mathcal{S}$, we have $w(x) \geq \widehat{V}(x, s)$

On the other hand, if we substitute $\tau^{*}=\inf \left\{t \geq 0 ; X_{t}<z^{*}\right\}$ for $\tau$ in (2.8)-(2.10), then all the inequalities are satisfied as equalities thanks to the assumptions (i)-(iv). Therefore, $w(x)=\widehat{V}(x, s)$ for every $x \leq s$.

To use Lemma 2.2, one usually constructs a candidate $w(\cdot)$ and proves the required inequalities (ii) and (iii). However, for the optimal stopping problems in spectrally negative Lévy models, this procedure is nontrivial and problem-specific, depending on various data such as functions $f, g, k$ and process $X$. It is because no general results about the optimality of threshold strategy have been proven. Accordingly, in this paper, having thus far characterized our two-dimensional problem (2.1) as a set of one-dimensional optimal stopping problems (2.6), we shall focus on and contribute to obtaining, in the general setting, an explicit form of solution (3.9) under threshold strategies. Note that, for our problem, optimality of (3.9) for (2.6) (and hence for (2.1)) is given by verifying the conditions in Lemma 2.2 with $K=V(s, s)$.

Recall that, in the linear diffusion case, a full characterization of the value function and of optimal stopping rule are known and the methodology for solution has been established; an optimal stopping rule is given by the threshold strategy in a very general setup. See Dayanik and Karatzas [11]; Propositions 5.7 and 5.14. See also Pham [24]; Section 5.2.3. Hence at least, if $X$ has no jump (that is, $X$ is Brownian motion with drift), the solution we derive is an optimal strategy for the function (2.1).

2.2. Scale functions. We review some mathematically important facts before solving the problem. Associated with every spectrally negative Lévy process, there exists a $(q-)$ scale function

$$
W^{(q)}: \mathbb{R} \mapsto \mathbb{R} ; \quad q \geq 0,
$$

that is continuous, strictly increasing on $[0, \infty)$ and 0 on $(-\infty, 0)$. It is uniquely determined by

$$
\int_{0}^{\infty} e^{-\beta x} W^{(q)}(x) \mathrm{d} x=\frac{1}{\psi(\beta)-q}, \quad \beta>\Phi(q) .
$$

Fix $a>x>0$ and define

$$
T_{a}:=\inf \left\{t \geq 0: X_{t}>a\right\} \quad \text { and } \quad T_{0}^{-}:=\inf \left\{t \geq 0: X_{t}<0\right\} .
$$

then we have

$$
\mathbb{E}^{x}\left[e^{-q T_{a}} 1_{\left\{T_{a}<T_{0}^{-}, T_{a}<\infty\right\}}\right]=\frac{W^{(q)}(x)}{W^{(q)}(a)}
$$

and

$$
\mathbb{E}^{x}\left[e^{-q T_{0}^{-}} 1_{\left\{T_{a}>T_{0}^{-}, T_{0}^{-}<\infty\right\}}\right]=Z^{(q)}(x)-Z^{(q)}(a) \frac{W^{(q)}(x)}{W^{(q)}(a)},
$$


where

$$
Z^{(q)}(x):=1+q \int_{0}^{x} W^{(q)}(y) \mathrm{d} y, \quad x \in \mathbb{R}
$$

Here we have $Z^{(q)}(x)=1$ on $(-\infty, 0]$. We also have

$$
\mathbb{E}^{x}\left[e^{-q T_{0}^{-}}\right]=Z^{(q)}(x)-\frac{q}{\Phi(q)} W^{(q)}(x), \quad x>0 .
$$

In particular, $W^{(q)}$ is continuously differentiable on $(0, \infty)$ if $\Pi$ does not have atoms and $W^{(q)}$ is twicedifferentiable on $(0, \infty)$ if $\sigma>0$; see, e.g., Chan et al.[9]. Throughout this paper, we assume the former:

Assumption 2.1. We assume that $\Pi$ does not have atoms.

Fix $q>0$. The scale function increases exponentially;

$$
W^{(q)}(x) \sim \frac{e^{\Phi(q) x}}{\psi^{\prime}(\Phi(q))} \quad \text { as } x \uparrow \infty .
$$

There exists a (scaled) version of the scale function $W_{\Phi(q)}=\left\{W_{\Phi(q)}(x) ; x \in \mathbb{R}\right\}$ that satisfies

$$
W_{\Phi(q)}(x)=e^{-\Phi(q) x} W^{(q)}(x), \quad x \in \mathbb{R}
$$

and

$$
\int_{0}^{\infty} e^{-\beta x} W_{\Phi(q)}(x) \mathrm{d} x=\frac{1}{\psi(\beta+\Phi(q))-q}, \quad \beta>0 .
$$

Moreover $W_{\Phi(q)}(x)$ is increasing, and as is clear from (2.15),

$$
W_{\Phi(q)}(x) \uparrow \frac{1}{\psi^{\prime}(\Phi(q))} \quad \text { as } x \uparrow \infty .
$$

Regarding its behavior in the neighborhood of zero, it is known that

$$
W^{(q)}(0)=\left\{\begin{array}{ll}
0, & \text { unbounded variation } \\
\frac{1}{d}, & \text { bounded variation }
\end{array}\right\},
$$

where $d:=\mu-\int_{(-1,0)} x \Pi(\mathrm{d} x)$, and

$$
W_{+}^{(q)^{\prime}}(0)=\left\{\begin{array}{ll}
\frac{2}{\sigma^{2}}, & \sigma>0 \\
\infty, & \sigma=0 \text { and } \Pi(0, \infty)=\infty \\
\frac{q+\Pi(0, \infty)}{d^{2}}, & \text { compound Poisson }
\end{array}\right\}
$$

see Lemmas 4.3-4.4 of Kyprianou and Surya [20]. For a comprehensive account of the scale function, see [7, 8, $18,20]$. See $[15,28]$ for numerical methods for computing the scale function. 


\section{EXPLiCIT SOLUTION}

Now we look to an explicit solution of $\bar{V}$ for $\tau \in \mathcal{S}^{\prime}$. Let us introduce the probability measure $\widetilde{\mathbb{P}}^{x, s}$ such that the Radon-Nikodym derivative between $\widetilde{\mathbb{P}}^{x, s}$ and $\mathbb{P}^{x, s}$ is defined by

$$
\left.\frac{\mathrm{d} \widetilde{\mathbb{P}}^{x, s}}{\mathrm{~d} \mathbb{P}^{x, s}}\right|_{\mathcal{F}_{t}}=e^{-q t+\Phi(q)\left(X_{t}-x\right)} .
$$

Under $\widetilde{\mathbb{P}}^{x, s}, X$ has the Laplace exponent $\widetilde{\psi}$ defined by

$$
\begin{aligned}
\widetilde{\psi}(\lambda)= & \psi(\lambda+\Phi(q))-\psi(\Phi(q)) \\
= & \left(\sigma^{2} \Phi(q)+\mu+\int_{(-\infty, 0)} x\left(e^{\Phi(q) x}-1\right) \mathbb{1}_{\{x>-1\}} \Pi(\mathrm{d} x)\right) \lambda \\
& +\frac{1}{2} \sigma^{2} \lambda^{2}+\int_{(-\infty, 0)}\left(e^{\lambda x}-1-\lambda x \mathbb{1}_{\{x>-1\}}\right) e^{\Phi(q) x} \Pi(\mathrm{d} x) .
\end{aligned}
$$

Note that since $\widetilde{\psi}^{\prime}(0+)=\psi^{\prime}(\Phi(q)+)>0, X$ drifts to $\infty$ for $q \geq 0$.

Let $W_{\Phi(q)}: \mathbb{R} \mapsto \mathbb{R}$ be the scale function of $X$ under $\widetilde{\mathbb{P}}^{x, s}$, that is, $W_{\Phi(q)}$ has the Laplace transform

$$
\int_{0}^{\infty} e^{-\lambda x} W_{\Phi(q)}(x) d x=\frac{1}{\widetilde{\psi}(\lambda)} .
$$

In addition, we define the process $\eta=\left\{\eta_{t} ; t \geq 0\right\}$ of the height of the excursion as

$$
\eta_{u}:=\sup \left\{(S-X)_{T_{u-}+w}: 0 \leq w \leq T_{u}-T_{u-}\right\}, \text { if } T_{u}>T_{u-},
$$

and $\eta_{u}=0$ otherwise, where $T_{u-}:=\inf \left\{t \geq 0: X_{t} \geq u\right\}=\lim _{m \rightarrow u-} T_{m}$. Then $\eta$ is a Poisson point process, and we denote its characteristic measure on $\widetilde{\mathbb{P}}^{x, s}$ by $\tilde{\nu}$. It is known that there is a relation between $W_{\Phi(q)}$ and $\tilde{\nu}$ :

$$
W_{\Phi(q)}(x)=c \exp \left(-\int_{x}^{\infty} \tilde{\nu}[u, \infty) \mathrm{d} u\right)
$$

where $c$ is some positive constant. See Bertoin [7] page 195 for the explanation of this identity.

If $\tau \in \mathcal{S}^{\prime}(b)$, equation (2.3) can be written as

$$
\begin{aligned}
& \mathbb{E}^{x, s}\left[\int_{0}^{\tau \wedge \zeta} e^{-q t} f\left(X_{t}, S_{t}\right) \mathrm{d} t\right] \\
= & \bar{f}(x, s)-\mathbb{E}^{x, s}\left[\mathbb{1}_{\{\tau<\zeta\}} e^{-q \tau} \bar{f}\left(X_{\tau}, S_{\tau}\right)+\mathbb{1}_{\{\tau=\zeta\}} e^{-q \tau} \bar{f}\left(X_{\tau}, S_{\tau}\right)\right] .
\end{aligned}
$$

Accordingly, the function $\bar{V}$ can be written as

$$
\bar{V}(x, s)=\bar{f}(x, s)+V(x, s)
$$

where

$$
V(x, s)=\sup _{\tau \in \mathcal{S}^{\prime}(b)} \mathbb{E}^{x, s}\left[\mathbb{1}_{\{\tau<\zeta\}} e^{-q \tau}(g-\bar{f})\left(X_{\tau}, S_{\tau}\right)-\mathbb{1}_{\{\tau=\zeta\}} e^{-q \tau}(k+\bar{f})\left(X_{\tau}, S_{\tau}\right)\right]
$$


3.1. When $X_{0}=S_{0}$. As a first step, we consider the case $X_{0}=S_{0}$. Set stopping times $T_{m}$ as $T_{m}=\inf \{t \geq 0$ : $\left.X_{t}>m\right\}$ (Recall (2.11)). From the strong Markov property of $(X, S)$, when $\tau(l) \in \mathcal{S}^{\prime}(b)$ and $S_{0}=X_{0}=s$, we have,

$$
\begin{aligned}
& \mathbb{E}^{s, s}\left[\mathbb{1}_{\{\tau(l)<\zeta\}} e^{-q \tau(l)}(g-\bar{f})\left(X_{\tau(l)}, S_{\tau(l)}\right)\right] \\
= & \int_{s}^{\infty} \mathbb{E}^{s, s}\left[\mathbb{1}_{\left\{\tau(l)<\zeta, S_{\tau(l)} \in \mathrm{d} m\right\}} e^{-q \tau(l)}(g-\bar{f})\left(X_{\tau(l)}, S_{\tau(l)}\right)\right] \\
= & \int_{s}^{\infty} \mathbb{E}^{s, s}\left[\mathbb { 1 } _ { \{ T _ { m } \leq \tau ( l ) \} } e ^ { - q T _ { m } } \mathbb { E } ^ { m , m } \left[e^{-q \tau_{l(m)}}(g-\bar{f})\left(X_{\tau_{l(m)}}, S_{\tau_{l(m)}}\right)\right.\right. \\
& \left.\left.\times \mathbb{1}_{\left\{S_{\tau_{l(m)}}-X_{\tau_{l(m)}} \leq b, S_{\tau_{l(m)}} \in \mathrm{d} m\right\}}\right]\right] \\
= & \int_{s}^{\infty} \mathbb{E}^{s, s}\left[\mathbb{1}_{\left\{S_{\tau(l)} \geq m\right\}} e^{-q T_{m}}\right]((g-\bar{f})(m-l(m), m) \\
& \times \mathbb{E}^{m, m}\left[e^{\left.-q \tau_{l(m)}\right)} \mathbb{1}_{\left\{Y_{\tau_{l(m)}}=l(m), S_{\tau_{l(m)}} \in \mathrm{d} m\right\}}\right] \\
& +\iint_{A}(g-\bar{f})(m-y+h, m) \\
& \left.\times \mathbb{E}^{m, m}\left[e^{-q \tau_{l(m)}} \mathbb{1}_{\left\{X_{\tau_{l(m)}}-X_{\tau_{l(m)}} \in \mathrm{d} h, S_{\tau_{l(m)}} \in \mathrm{d} m, Y_{\tau_{l(m)}} \in \mathrm{d} y\right\}}\right]\right),
\end{aligned}
$$

where

$$
A=\left\{(y, h) \in \mathbb{R}^{2} ; y-h \in[l(m), b], h<0, y \in[0, l(m)]\right\} .
$$

Now we examine each term in the last line of (3.2). Since $X$ is a spectrally negative process and $S$ is its running maximum process, by (3.1) we have, for $m \geq s$,

$$
\begin{aligned}
\mathbb{E}^{s, s}\left[\mathbb{1}_{\left\{S_{\tau(l)} \geq m\right\}} e^{-q T_{m}}\right] & =\widetilde{\mathbb{E}}^{s, s}\left[e^{-(m-s) \Phi(q)} \mathbb{1}_{\left\{S_{\tau(l)} \geq m\right\}}\right] \\
& =e^{-(m-s) \Phi(q) \widetilde{\mathbb{P}}^{s, s}\left(S_{\tau(l)} \geq m\right)} \\
& =\exp \left(-\int_{s}^{m}\left(\frac{W_{\Phi(q)}^{\prime}(l(u)+)}{W_{\Phi(q)}(l(u))}+\Phi(q)\right) \mathrm{d} u\right) \\
& =\exp \left(-\int_{s}^{m} \frac{W_{+}^{(q)^{\prime}}(l(u))}{W^{(q)}(l(u))} \mathrm{d} u\right) .
\end{aligned}
$$

From Theorems 1 and 2 in Pistorius [26], we have

$$
\begin{gathered}
\mathbb{E}^{m, m} \quad\left[e^{-q \tau_{l(m)}} \mathbb{1}_{\left\{X_{\tau_{l(m)}}-X_{\tau_{l(m)}-} \in \mathrm{d} h, S_{\tau_{l(m)}} \in \mathrm{d} m, Y_{\tau_{l(m)}} \in \mathrm{d} y\right\}}\right] \\
=\mathbb{1}_{\{y-h>l(m)\}} \Pi(\mathrm{d} h)\left(W_{+}^{(q)^{\prime}}(y)-\frac{W_{+}^{(q)^{\prime}}(l(m))}{W^{(q)}(l(m))} W^{(q)}(y)\right) \mathrm{d} y \mathrm{~d} m,
\end{gathered}
$$

and

$$
\mathbb{E}^{m, m}\left[e^{-q \tau_{l(m)}} \mathbb{1}_{\left\{Y_{\tau_{l(m)}}=l(m), S_{\tau_{l(m)}} \in \mathrm{d} m\right\}}\right]=\frac{\sigma^{2}}{2}\left(\frac{W_{+}^{(q)^{\prime}}(l(m))^{2}}{W^{(q)}(l(m))}-W_{+}^{(q)^{\prime \prime}}(l(m))\right) .
$$


Putting together, if $\tau(l) \in \mathcal{S}^{\prime}(b),(3.2)$ becomes

$$
\begin{aligned}
& \mathbb{E}^{s, s}\left[\mathbb{1}_{\left\{\tau(l)<\zeta, S_{\tau(l)} \in \mathrm{d} m\right\}} e^{-q \tau(l)}(g-\bar{f})\left(X_{\tau(l)}, S_{\tau(l)}\right)\right] \\
= & \exp \left(-\int_{s}^{m} \frac{W_{+}^{(q)^{\prime}}(l(u))}{W^{(q)}(l(u))} \mathrm{d} u\right)\left(\frac{\sigma^{2}}{2}\left(\frac{W_{+}^{(q)^{\prime}}(l(m))^{2}}{W^{(q)}(l(m))}-W_{+}^{(q)^{\prime \prime}}(l(m))\right)\right. \\
& \times(g-\bar{f})(m-l(m), m)+\int_{0}^{l(m)} \mathrm{d} y \int_{y-b}^{y-l(m)} \Pi(\mathrm{d} h)(g-\bar{f})(m-y+h, m) \\
& \left.\times\left(W_{+}^{(q)^{\prime}}(y)-\frac{W_{+}^{(q)^{\prime}}(l(m))}{W^{(q)}(l(m))} W^{(q)}(y)\right)\right) \mathrm{d} m .
\end{aligned}
$$

In the same way as above, if $\tau(l) \in \mathcal{S}^{\prime}(b)$, we obtain for the second term of the expectation in (3.2)

$$
\begin{aligned}
& \mathbb{E}^{x, s}\left[\mathbb{1}_{\left\{\tau(l)=\zeta, S_{\tau(l)} \in \mathrm{d} m\right\}} e^{-q \tau(l)}(\bar{f}+k)\left(X_{\tau(l)}, S_{\tau(l)}\right)\right] \\
= & \int_{s}^{\infty} \mathbb{E}^{s, s}\left[\mathbb { 1 } _ { \{ T _ { m } \leq \tau ( l ) \} } e ^ { - q T _ { m } } \mathbb { E } ^ { m , m } \left[e^{-q \tau_{l(m)}(\bar{f}+k)\left(X_{\tau_{l(m)}}, S_{\tau_{l(m)}}\right)}\right.\right. \\
& \left.\times \mathbb{1}_{\left.\left\{S_{\tau_{l(m)}}-X_{\tau_{l(m)}}>b, S_{\tau_{l(m)}} \in \mathrm{d} m\right\}\right]}\right] \\
= & \exp \left(-\int_{s}^{m} \frac{W_{+}^{(q)^{\prime}}(l(u))}{W^{(q)}(l(u))} \mathrm{d} u\right) \iint_{B}(\bar{f}+k)(m-y+h, m) \\
= & \quad \exp \left(-\int_{s}^{m, m} \frac{W_{+}^{(q)^{\prime}}(l(u))}{W^{(q)}(l(u))} \mathrm{d} u\right)\left(e_{0}^{\left.-q \tau_{l(m)} \mathbb{1}_{\left\{X_{\tau_{l(m)}}\right.}-X_{\tau_{l(m)}} \in \mathrm{d} h, S_{\tau_{l(m)}} \in \mathrm{d} m, Y_{\tau_{l(m)}} \in \mathrm{d} y\right\}}\right] \\
& \times(\bar{f}+k)\left(m-y \int_{-\infty}^{y-b} \Pi(\mathrm{d} h)\right. \\
&
\end{aligned}
$$

where

$$
B=\left\{(y, h) \in \mathbb{R}^{2} ; y-h>b, h<0, y \in[0, l(m)]\right\} .
$$

For notational simplicity, let the function $F_{m}(z):[0, b] \mapsto \mathbb{R}$ defined by

$$
\begin{aligned}
F_{m}(z):= & \frac{\sigma^{2}}{2}\left(\frac{W_{+}^{(q)^{\prime}}(z)^{2}}{W^{(q)}(z)}-W_{+}^{(q)^{\prime \prime}}(z)\right)(g-\bar{f})(m-z, m) \\
& +\int_{0}^{z} \mathrm{~d} y \int_{y-b}^{y-z} \Pi(\mathrm{d} h)(g-\bar{f})(m-y+h, m) \\
& \times\left(W_{+}^{(q)^{\prime}}(y)-\frac{W_{+}^{(q)^{\prime}}(z)}{W^{(q)}(z)} W^{(q)}(y)\right) \\
& -\int_{0}^{z} \mathrm{~d} y \int_{-\infty}^{y-b} \Pi(\mathrm{d} h)(\bar{f}+k)(m-y+h, m) \\
& \times\left(W_{+}^{(q)^{\prime}}(y)-\frac{W_{+}^{(q)^{\prime}}(z)}{W^{(q)}(z)} W^{(q)}(y)\right) .
\end{aligned}
$$


Hence we have, up to this point, proved the following:

Proposition 3.1. When $X_{0}=S_{0}$, the function $V(s, s)$ for $\tau \in \mathcal{S}^{\prime}$ can be represented by

$$
V(s, s)=\sup _{l} \int_{s}^{\infty} \exp \left(-\int_{s}^{m} \frac{W^{(q)}(l(u))}{W_{+}^{(q)^{\prime}}(l(u))} \mathrm{d} u\right) F_{m}(l(m)) \mathrm{d} m
$$

where $F_{m}(\cdot)$ is defined in (3.4)

Recall that $l(s)$ denotes the height of the excursion $Y=S-X$ when $S=s$. We wish to find, given $s$, the optimal height $l^{*}(s)$ to stop the process, and to calculate $V(s, s)$ explicitly.

Proposition 3.2. Under $q \geq 0$ and $\sigma>0$ instead of Assumption 2.1, suppose further that $F_{s}: \mathbb{R}_{+} \mapsto \mathbb{R}$ is continuous. Then we have

$$
V(s, s)=\frac{F_{s}\left(l^{*}(s)\right) W^{(q)}\left(l^{*}(s)\right)}{W_{+}^{(q)^{\prime}}\left(l^{*}(s)\right)},
$$

and $l^{*}(s)$ is the maximizer of the map $z \mapsto \frac{F_{s}(z) W^{(q)}(z)}{W_{+}^{(q)^{\prime}}(z)}$ on $[0, b]$.

Remark 3.1. (i) Note that the maximizer $l^{*}(s)$ exists on $[0, b]$ since the map $z \mapsto F_{s}(z) W^{(q)}(z) / W_{+}^{(q)^{\prime}}(z)$ is continuous due to $W^{(q)} \in C^{2}$ (by $\sigma>0$ ) and $[0, b]$ is compact.

(ii) If $q>0$, a sufficient condition for the continuity of $F_{s}$ is the continuity of $f, g$ and $k$. This is a consequence of the continuity of $x \mapsto \mathbb{E}^{x, s}\left[f\left(X_{t}, S_{t}\right)\right]$ for all $t \geq 0$ and $s \in \mathbb{R}_{+}, W^{(q)} \in C^{2}$ and

$$
|\bar{f}(x, s)-\bar{f}(y, s)| \leq q^{-1}|f(x, s)-f(y, s)|
$$

for all $x, y \in \mathbb{R}$. If $q=0$, sufficient conditions for the continuity of $F_{s}$ are more restrictive, for example, the boundedness of $f$.

Proof. From the equation (3.5), we have for any $\epsilon>0$,

$$
\begin{aligned}
V(s, s)= & \sup _{l}\left[\exp \left(-\int_{s}^{s+\epsilon} \frac{W^{(q)}(l(u))}{W_{+}^{(q)^{\prime}}(l(u))} \mathrm{d} u\right)\right. \\
& \times \int_{s+\epsilon}^{\infty} \exp \left(-\int_{s+\epsilon}^{m} \frac{W^{(q)}(l(u))}{W_{+}^{(q)^{\prime}}(l(u))} \mathrm{d} u\right) F_{m}(l(m)) \mathrm{d} m \\
& \left.+\int_{s}^{s+\epsilon} \exp \left(-\int_{s}^{m} \frac{W^{(q)}(l(u))}{W_{+}^{(q)}(l(u))} \mathrm{d} u\right) F_{m}(l(m)) \mathrm{d} m\right] \\
= & \sup _{l}\left[\exp \left(-\int_{s}^{s+\epsilon} \frac{W^{(q)}(l(u))}{W_{+}^{(q)^{\prime}}(l(u))} \mathrm{d} u\right) V(s+\epsilon, s+\epsilon)\right. \\
& \left.+\int_{s}^{s+\epsilon} \exp \left(-\int_{s}^{m} \frac{W^{(q)}(l(u))}{W_{+}^{(q)}(l(u))} \mathrm{d} u\right) F_{m}(l(m)) \mathrm{d} m\right]
\end{aligned}
$$

This expression motivates us to set $V_{\epsilon}: \mathbb{R} \mapsto \mathbb{R}$ as

$$
V_{\epsilon}(s):=\sup _{l(s)}\left[\exp \left(-\frac{\epsilon W^{(q)}(l(s))}{W_{+}^{(q)^{\prime}}(l(s))}\right) V(s+\epsilon, s+\epsilon)+\epsilon F_{s}(l(s))\right] .
$$


Then we have $\lim _{\epsilon \downarrow 0} V_{\epsilon}(s)=V(s, s)$. Since $\lim _{\epsilon \downarrow 0} V(s+\epsilon, s+\epsilon)=V(s, s)$, the optimal threshold $l^{*}(s)$ should satisfy

$$
\lim _{\epsilon \downarrow 0} V_{\epsilon}(s)=\lim _{\epsilon \downarrow 0}\left[\exp \left(-\frac{\epsilon W^{(q)}\left(l^{*}(s)\right)}{W_{+}^{(q)^{\prime}}\left(l^{*}(s)\right)}\right) V(s+\epsilon, s+\epsilon)+\epsilon F_{s}\left(l^{*}(s)\right)\right] .
$$

From this equation, we obtain

$$
\begin{aligned}
V(s, s) & =\lim _{\epsilon \downarrow 0} \frac{V_{\epsilon}(s)-\exp \left(-\frac{\epsilon W_{+}^{(q)^{\prime}}\left(l^{*}(s)\right)}{W^{(q)}\left(l^{*}(s)\right)}\right) V(s+\epsilon, s+\epsilon)}{\left(1-\exp \left(-\frac{\epsilon W_{+}^{(q)^{\prime}}\left(l^{*}(s)\right)}{W^{(q)}\left(l^{*}(s)\right)}\right)\right)} \\
& =\lim _{\epsilon \downarrow 0} \frac{\epsilon F_{s}\left(l^{*}(s)\right)}{\left(1-\exp \left(-\frac{\epsilon W_{+}^{(q)^{\prime}}\left(l^{*}(s)\right)}{W^{(q)}\left(l^{*}(s)\right)}\right)\right.}=\frac{F_{s}\left(l^{*}(s)\right) W^{(q)}\left(l^{*}(s)\right)}{W_{+}^{(q)^{\prime}}\left(l^{*}(s)\right)}
\end{aligned}
$$

where the last equality is obtained by L'Hôpital's rule, and hence $l^{*}(s)$ is the value which gives supremum to $\frac{F_{s}(z) W^{(q)}(z)}{W_{+}^{(q)^{\prime}}(z)}$.

Remark 3.2. $\frac{F_{s}(z) W^{(q)}(z)}{W_{+}^{()^{\prime}}(z)}$ is the value for the strategy $l$ with $l(s)=z$ and $l=l^{*}$ for every $m>s$; that is, this amount is obtained when we stop if $X$ goes below $s-z$ in the excursion at level $S=s$ and use optimal strategy for the higher level $S>s$.

3.2. When $S_{0}>X_{0}$. Finally, let us consider the case of $S_{0}>X_{0}$. In this case, $\bar{V}$ can be represented in terms of $\bar{V}(s, s)$ as follows:

$$
\begin{aligned}
\bar{V}(x, s)= & \bar{f}(x, s)+\sup _{\tau \in \mathcal{S}^{\prime}(b)} \mathbb{E}^{x, s}\left[\mathbb{1}_{\left\{T_{s}<\tau\right\}} e^{-q T_{s}}(\bar{V}-\bar{f})(s, s)\right. \\
& +\mathbb{1}_{\left\{\tau<T_{s} \wedge \zeta\right\}} e^{-q \tau}(g-\bar{f})\left(X_{\tau}, s\right) \\
& \left.-\mathbb{1}_{\left\{\zeta=\tau<T_{s}\right\}} e^{-q \tau}(k+\bar{f})\left(X_{\tau}, s\right)\right]
\end{aligned}
$$

Set $\tau=\tau(l)$. Then, from (2.12), the first term in (3.8) can be written by

$$
\mathbb{E}^{x, s}\left[\mathbb{1}_{\left\{T_{s}<\tau\right\}} e^{-q T_{s}}(\bar{V}-\bar{f})(s, s)\right]=\frac{W^{(q)}(l(s)+x-s)}{W^{(q)}(l(s))}(\bar{V}-\bar{f})(s, s) .
$$


For the second term, we use Theorem 1 and 2 in Pistorius[26] again to obtain, for $x \in[s-l(s), s]$

$$
\begin{aligned}
& \mathbb{E}^{x, s}\left[\mathbb{1}_{\left\{\tau<T_{s} \wedge \zeta\right\}} e^{-q \tau}(g-\bar{f})\left(X_{\tau}, s\right)\right] \\
& =\mathbb{E}^{x, s}\left[e^{-q \tau_{l(s)}} \mathbb{1}_{\left\{Y_{\tau_{l(s)}}=l(s), S_{\tau_{l(s)}}=s\right\}}\right](g-\bar{f})(s-l(s), s) \\
& +\iint_{A}(g-\bar{f})(s-y+h, s) \mathbb{E}^{x, s}\left[e^{-q \tau_{l(s)}} \mathbb{1}_{\left\{X_{\tau_{l(s)}}-X_{\tau_{l(s)}} \in \mathrm{d} h, S_{\tau_{l(s)}}=s, Y_{\tau_{l(s)}} \in \mathrm{d} y\right\}}\right] \\
& =\frac{\sigma^{2}}{2}\left(W_{+}^{(q)^{\prime}}(l(s)+x-s)-\frac{W_{+}^{(q)^{\prime}}(l(s))}{W^{(q)}(l(s))} W^{(q)}(l(s)+x-s)\right) \\
& \times(g-\bar{f})(s-l(s), s)+\int_{0}^{l(s)} \mathrm{d} y \int_{y-b}^{y-l(s)} \Pi(\mathrm{d} h)(g-\bar{f})(s-y+h, s) \\
& \times\left(\frac{W^{(q)}(l(s)+x-s)}{W^{(q)}(l(s))} W^{(q)}(y)-W^{(q)}(y+x-s)\right) .
\end{aligned}
$$

For the third term, we have, in the same way as above,

$$
\begin{aligned}
& \mathbb{E}^{x, s}\left[\mathbb{1}_{\left\{\zeta=\tau<T_{s}\right\}} e^{-q \tau}(k+\bar{f})\left(X_{\tau}, s\right)\right] \\
= & \iint_{B}(k+\bar{f})(s-y+h, s) \mathbb{E}^{x, s}\left[e^{-q \tau_{l(s)}} \mathbb{1}_{\left\{X_{\tau_{l(s)}}-X_{\tau_{l(s)}-} \in \mathrm{d} h, S_{\tau_{l(s)}}=s, Y_{\tau_{l(s)}} \in \mathrm{d} y\right\}}\right] \\
= & \int_{0}^{l(s)} \mathrm{d} y \int_{-\infty}^{y-b} \Pi(\mathrm{d} h)(k+\bar{f})(s-y+h, s) \\
& \times\left(\frac{W^{(q)}(l(s)+x-s)}{W^{(q)}(l(s))} W^{(q)}(y)-W^{(q)}(y+x-s)\right)
\end{aligned}
$$

for $x \in[s-l(s), s]$.

Now by combining all these terms, we can write $\bar{V}(x, s)$ for $x \in\left[s-l^{*}(s), s\right]$,

$$
\begin{aligned}
\bar{V}(x, s) & =\bar{f}(x, s)+\frac{W^{(q)}\left(l^{*}(s)+x-s\right)}{W^{(q)}\left(l^{*}(s)\right)}(\bar{V}-\bar{f})(s, s) \\
& +\frac{\sigma^{2}}{2}(g-\bar{f})\left(s-l^{*}(s), s\right) \\
& \times\left(W_{+}^{(q)^{\prime}}\left(l^{*}(s)+x-s\right)-\frac{W_{+}^{(q)^{\prime}}\left(l^{*}(s)\right)}{W^{(q)}\left(l^{*}(s)\right)} W^{(q)}\left(l^{*}(s)+x-s\right)\right) \\
& +\int_{0}^{l^{*}(s)} \mathrm{d} y \int_{y-b}^{y-l^{*}(s)} \Pi(\mathrm{d} h)(g-\bar{f})(s-y+h, s) \\
& \times\left(\frac{W^{(q)}\left(l^{*}(s)+x-s\right)}{W^{(q)}\left(l^{*}(s)\right)} W^{(q)}(y)-W^{(q)}(y+x-s)\right) \\
& -\int_{0}^{l^{*}(s)} \mathrm{d} y \int_{-\infty}^{y-b} \Pi(\mathrm{d} h)(k+\bar{f})(s-y+h, s) \\
& \left(\frac{W^{(q)}\left(l^{*}(s)+x-s\right)}{W^{(q)}\left(l^{*}(s)\right)} W^{(q)}(y)-W^{(q)}(y+x-s)\right)
\end{aligned}
$$




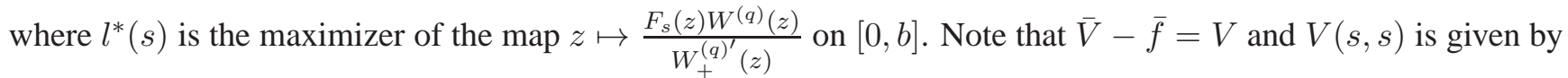
(3.6) (or (3.5)). For the stopping regions, it becomes from (3.8)

$$
\begin{gathered}
\bar{V}(x, s)=g(x, s), \quad x \in\left[s-b, s-l^{*}(s)\right] \\
\bar{V}(x, s)=-k(x, s), \quad x \in(-\infty, s-b) .
\end{gathered}
$$

\section{BANK’s Optimization Under CAPITAL REQUiREMENTS}

In this section, we solve an example. Imagine that a bank's total asset value is represented by $e^{X}$. We set that the leverage ratio, defined as (Debt)/(Total Asset), cannot exceed $e^{-b}$. For example, if the bank has the initial asset of $e^{x}=100$ with $e^{-b}=0.8$, it has total asset of 100 financed by debt 80 and equity 20 . We can think of this ratio as the maximum leverage ratio that is allowed by the banking regulations. We assume that the bank increases its asset base as long as $X=S$ where $S$ is the running maximum of $X$ and that the bank's leverage ratio is maintained at 0.8 . Hence if the asset value appreciates to 120 , then this would provide the bank with more lending opportunity since the equity value is now 40 . With this new equity level, the bank increases its leverage up to 0.8 , that is, total asset increasing to 200 financed by debt 160 and equity 40 . Note that $e^{S}=e^{X}=200$ and the debt level is $e^{-b} e^{S}=e^{S-b}=160$. Now if the bank's asset deteriorates due to defaults in the lending portfolio, we would have $S-X>0$. In other words, there appears an excursion from the level of $e^{S}=200$. Since the asset level has been pegged at $e^{S}=200$, the bank's equity would be wiped out when $e^{S-b}=e^{X}$. That is, when $e^{X}=160$ and the process is absorbed.

This model well describes a real situation where even a large bank can fail easily as we have experienced several times, the recent and magnified shock being the global financial crisis in 2007-2008. After becoming a large bank, it may still have an incentive to increase assets, seeking for profits. The danger of becoming insolvent is still $X=S-b$ if the bank continues to use leverage ratio of $e^{-b}$. The absorbing boundary is always coming after.

Moreover, note that this model can incorporate the regulatory requirements that the bank, when experiencing asset deterioration, need to sell the assets in order to reduce the leverage. ${ }^{1}$ For example, assume that when the bank loses one dollar of asset, the bank loses its equity by $\alpha$ and reduces its debt by $1-\alpha$, where $\alpha \in(0,1]$. Then, at the time the equity is wiped out, we have

$$
e^{X} \leq e^{S}\left(1-\frac{1-e^{-b}}{\alpha}\right)
$$

that is, the process is absorbed when the excursion $S-X$ reaches $-\log \left(1-\frac{1-e^{-b}}{\alpha}\right)$. Note that an application of this setting to bank regulations is studied in Egami and Oryu [14].

We consider the problem (2.1) with the process

$$
X_{t}=x+\mu t+\sigma B_{t}+\sum_{i=1}^{N(t)} \xi_{i},
$$

where $B$ is a standard Brownian motion, $N$ is a Poisson process with intensity $a$, and $\xi_{i}(i=1,2, \ldots)$ are independent identically distributed random variables whose distributions are exponential with parameter $\rho$ under $\mathbb{P}$. Note

\footnotetext{
${ }^{1}$ We are thankful to Nan Chen for pointing out this requirement.
} 
that if we put $a=0$, then $X$ is a Brownian motion with drift. The reward functions are set by $f(x, s)=e^{x / 2}$, $g(x, s)=e^{x}$, and $k(x, s)=0$, so that the problem is $V(x, s)=\sup _{\tau \in \mathcal{S}} \mathbb{E}^{x, s}\left[\int_{0}^{\tau \wedge \zeta} e^{-q t}\left(e^{X_{t} / 2}\right) \mathrm{d} t+\mathbb{1}_{\{\tau<\zeta\}} e^{-q \tau} e^{X_{\tau}}\right]$.

In this case, the Laplace exponent $\psi$ of $X$ is given by

$$
\psi(\lambda)=\mu \lambda+\frac{\sigma^{2} \lambda^{2}}{2}-\frac{a \lambda}{\rho+\lambda} .
$$

$\psi(\lambda)=q$ has three solutions $\Phi(q), \alpha$, and $\beta$ (in decreasing order) and $q$-scale function $W^{(q)}$ of $X$ is represented with these values;

$$
W^{(q)}(x)=\frac{e^{\Phi(q) x}}{\psi^{\prime}(\Phi(q))}+\frac{e^{\alpha x}}{\psi^{\prime}(\alpha)}+\frac{e^{\beta x}}{\psi^{\prime}(\beta)} .
$$

In the Brownian motion case, $\psi(\lambda)=q$ has only two solutions $\Phi(q)$ and $\alpha$, and the third term above vanishes.

For the existence of $\bar{f}$, we need some restriction on the parameters. $\bar{f}$ can be calculated by

$$
\begin{aligned}
\bar{f}(x, s) & =\mathbb{E}^{x, s}\left[\int_{0}^{\infty} e^{-q t} f\left(X_{t}, S_{t}\right) \mathrm{d} t\right] \\
& =\int_{0}^{\infty} e^{-q t} \mathbb{E}^{x, s}\left[f\left(X_{t}, S_{t}\right)\right] \mathrm{d} t \\
& =e^{\frac{x}{2}} \int_{0}^{\infty} e^{\left(\psi\left(\frac{1}{2}\right)-q\right) t} \mathrm{~d} t .
\end{aligned}
$$

Hence the condition we need is $\psi\left(\frac{1}{2}\right)-q=\frac{\mu}{2}+\frac{\sigma^{2}}{8}-\frac{a}{2 \rho+1}-q<0$, and then

$$
\bar{f}(x, s)=-\frac{e^{\frac{x}{2}}}{\frac{\mu}{2}+\frac{\sigma^{2}}{8}-\frac{a}{2 \rho+1}-q} .
$$

4.1. Brownian motion. First, we consider a Brownian motion with drift by using parameters $\mu=0.05, \sigma=0.1$, $a=0, q=0.1$, and $b=1$. Note that the value of $\rho$ has no effects on the problem when $a=0$.

Panels (i), (ii), and (iii) in Figure 1 are the graphs of $\frac{F_{s}(z) W^{(q)}(z)}{W_{+}^{()^{\prime}}(z)}$ with $s=5,5.2141$, and 5.3, respectively. As shown in Proposition 3.2, the maximum value in each graph corresponds to $V(s, s)$ for various values of $s$, and $z=l^{*}(s)$ are the maximizers. In the case (i) $s=5$, we have the boundary solution $l(5)=1$. This means that, in

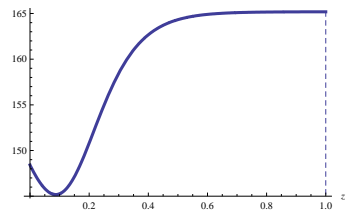

(i) $\mathrm{s}=5$

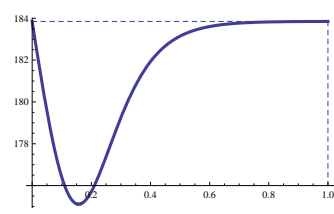

(ii) $\mathrm{s}=5.2141$

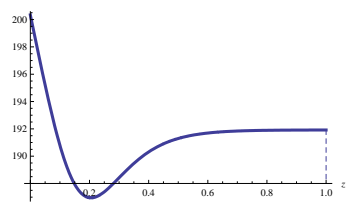

(iii) $\mathrm{s}=5.3$

FIGURE 1. Graphs of $\frac{F_{s}(z) W^{(q)}(z)}{W_{+}^{(q)^{\prime}}(z)}$ for various values of $s$.

the excursion which occurs at level $S=5$, it is optimal to stop when $X$ goes below $5-l(5)=4$. Since we set $b=1$ here, if $X$ creeps over the level 4 , we can obtain the terminal reward. The case (ii) $s=5.2141$ is a special point in some sense. Unlike the case $s<5.2141$, there are two solutions $l(5.2141)=0$ and 1 . If we choose the former strategy $l(5.2141)=0$, this means, when $X$ reaches level 5.2141 for the first time, we stop it immediately 
and gain the terminal reward. If we choose the latter one $l(5.2141)=1$, that means we should stop when $X$ goes below $5.2141-l(5.2141)=4.2141$. In the case (iii), there is the boundary solution $l(5.3)=0$. That is, when $X$ reaches level 5.3 for the first time, we should exit immediately and gain the terminal reward.

These arguments are summarized in Figure 2 that illustrates an optimal strategy $l$ over the whole region of $s \in \mathbb{R}_{+}$. The dashed line is drawn at $s=5.2141$, which indicates the turning points of strategies. For $s<5.2141$, $l$ constantly takes the value of 1 . In this region, it is optimal to stop when the height of the excursion is 1 . For $s>5.2141, l$ constantly takes on 0 . In this region, our strategy reduces to the classical threshold strategy by observing the path of process $X$. That is, stop at the first passage time of level 5.2141 by the process $X$.

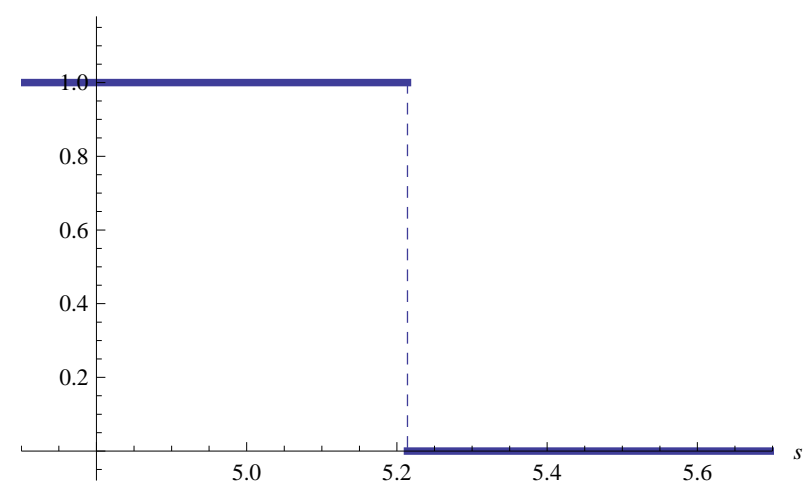

FIGURE 2. Graph of an optimal strategy $l^{*}$.

4.2. Brownian motion with exponential jumps. Next, to see the effects of jumps on the threshold strategy, we set $\mu=0.25, \sigma=0.1, a=2, \rho=10, q=0.1$, and $b=1$. Panels (i), (ii), (iii), and (iv) in Figure 4 are the graphs of $\frac{F_{s}(z) W^{(q)}(z)}{W_{+}^{()^{\prime}}(z)}$ with $s=4,5,5.1963$ and 5.3, respectively. Note that the number $d$ in (2.18) is 0.05 which is equal to the drift of the Brownian motion in Section 4.1.

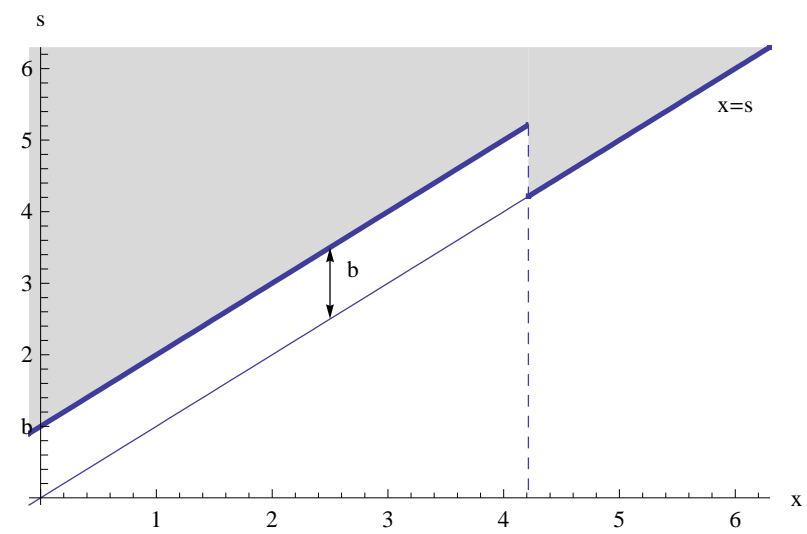

FIGURE 3. Graph of an optimal strategy $l^{*}$ on $(x, s)$-plane. The shaded area is optimal stopping region. 


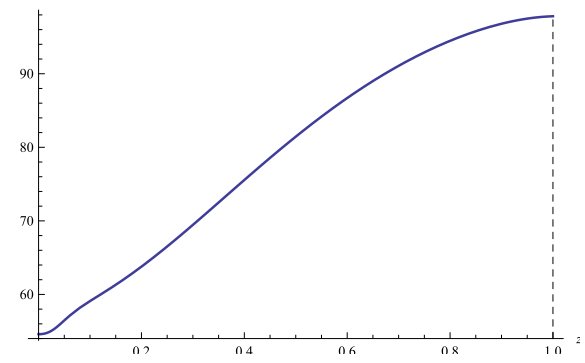

(i) $\mathrm{s}=4$

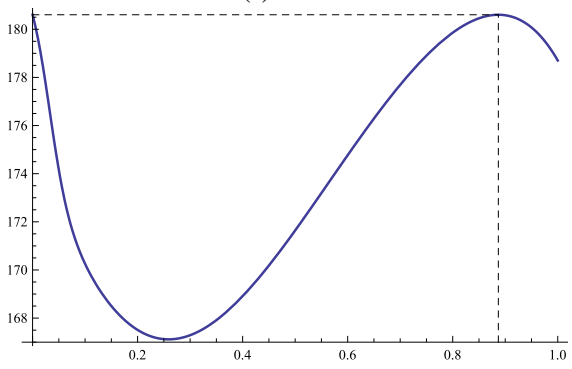

(iii) $\mathrm{s}=5.1963$

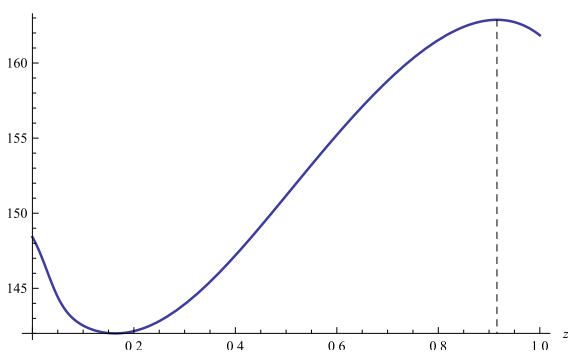

(ii) $\mathrm{s}=5$

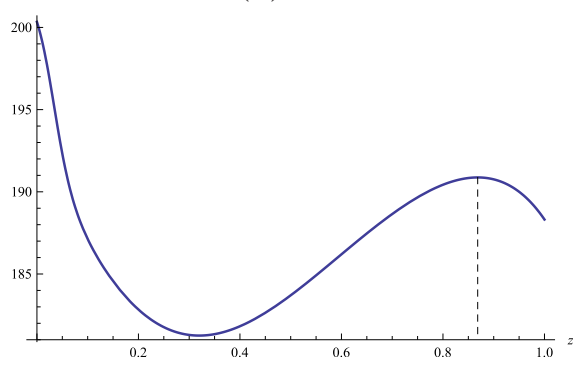

(iv) $\mathrm{s}=5.3$

FIGURE 4. Graphs of $\frac{F_{s}(z) W^{(q)}(z)}{W_{+}^{(q)^{\prime}}(z)}$ for various values of $s$.

In the case (i) $s=4$, we have the boundary solution $l(4)=1$. This means that, in the excursion which occurs at level $S=4$, it is optimal to stop when $X$ goes below $4-l(4)=3$. Since we set $b=1$ here, if $X$ creeps over the level 3, we can obtain the terminal reward. Instead, if $X$ jumps over the level 3 , we should pay the penalty (but we set this as 0 here) and cannot gain the terminal reward. In the case (ii) $s=5$, there is the internal solution $l(5)=0.915551<1=b$. Therefore, in the excursion which occurs at level $S=5$, it is optimal to stop immediately that $X$ goes below $5-l(5)=4.08445$. Since $l(5)<b$, if $X$ creeps over the level $5-l(5)$ or jumps onto in the area between $5-l(5)$ and $5-b=4$, we can obtain the terminal reward. But if $X$ jumps across the level 4 , we cannot obtain the terminal reward. The case (iii) $s=5.1963$ is a turning point of strategy like the case (ii)

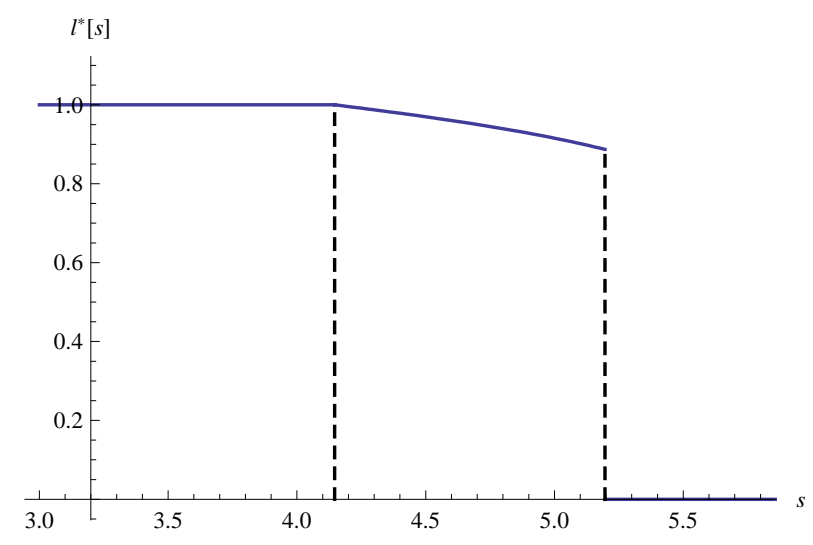

FIGURE 5. Graph of an optimal strategy $l^{*}$. 


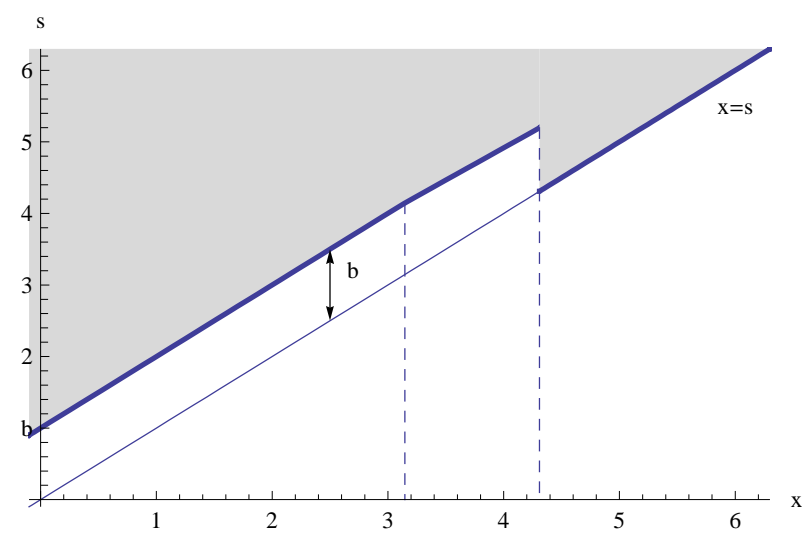

FIGURE 6. Graph of an optimal strategy $l^{*}$ on $(x, s)$-plane. The shaded area is optimal stopping region.

in Figure 1. There are two solutions $l(5.1963)=0$ and 0.886898. If we choose the former strategy $l(5.1963)=0$, this means, when $X$ reaches level 5.1963 for the first time, we stop it immediately and gain the terminal reward. If we choose the latter one $l(5.1963)=0.886898$, that means we should behave like the case (ii). In the case (iv), there is the boundary solution $l(5.3)=0$. That is, when $X$ reaches level 5.3 for the first time, we should exit immediately and gain the terminal reward.

These arguments are summarized in Figure 5 that illustrates an optimal strategy $l$ over the whole region of $s \in \mathbb{R}_{+}$. Two dashed lines are drawn at $s=4.1464$ and $s=5.1963$, which indicate the turning points of the strategies. For $s<4.1464, l$ constantly takes the value of 1 . In this region, it is optimal to stop when the height of the excursion is 1 . When $s$ lies between 4.1464 and $5.1963, l$ has the form of concave curve started at 1 . In this region, one should stop once the height of excursion is greater than or equal to $l(s)<1$. Note that this region does not show up in the Brownian motion case. Finally, for $s>5.1963, l$ constantly takes on 0 . In this region, our strategy reduces to the classical threshold strategy by observing the path of process $X$. That is, stop at the first passage time of level 5.1963 by the process $X$.

Before concluding this section, let us make some comparisons of the two cases. In the jump case, we have two levels $s^{1}:=4.1464$ and $s^{2}:=5.1963$ that mark the change points of strategy, while we have only one point $s^{0}:=5.2141$ in the no jump case. At the level of $s \in\left(s^{1}, s^{2}\right)$, the bank should stop the process during the excursion, a strategy that does not exist in the no jump case. It is due to the existence of jumps which could bring the process suddenly to the ruin. We have $s_{2}<s_{0}$, which again shows that jumps make the bank more cautious; with $(a, 1 / \rho)=(2,0.1)$ it should not expect to reach a level as high as $s_{0}$ even with the large $\mu=0.25$.

\section{REFERENCES}

[1] L. Alili and A. E. Kyprianou. Some remarks on first passage of Lévy processes, the American put and smooth pasting. Ann. Appl. Probab., 15:2062-2080, 2004.

[2] L. H. R. Alvarez. On the properties of r-excessive mappings for a class of diffusions. Ann. Appl. Probab., 13 (4):1517$1533,2003$. 
[3] F. Avram, A. E. Kyprianou, and M. R. Pistorius. Exit problems for spectrally negative Lévy processes and applications to (Canadized) Russion options. Ann. Appl. Probab., 14:215-235, 2004.

[4] F. Avram, Z. Palmowski, and M. R. Pistorius. On the optimal dividend problem for a spectrally negative Lévy process. Ann. Appl. Probab., 17(1):156-180, 2007.

[5] E. Baurdoux and A. E. Kyprianou. The McKean stochastic game driven by a spectrally negative Lévy process. Electron. J. Probab., 13:no. 8, 173-197, 2008.

[6] E. Baurdoux and A. E. Kyprianou. The Shepp-Shiryaev stochastic game driven by a spectrally negative Lévy process. Theory Probab. Appl., 53, 2009.

[7] J. Bertoin. Lévy processes, volume 121 of Cambridge Tracts in Mathematics. Cambridge University Press, Cambridge, 1996.

[8] J. Bertoin. Exponential decay and ergodicity of completely asymmetric Lévy processes in a finite interval. Ann. Appl. Probab., 7(1):156-169, 1997.

[9] T. Chan, A.E. Kyprianou, and M. Savov. Smoothness of scale functions for spectrally negative Lévy processes. Probab. Theory Relat. Fields, to appear.

[10] R. Cont and P. Tankov. Financial modelling with jump processes. Chapman Hall/CRC, Boca Raton, 2004.

[11] S. Dayanik and I. Karatzas. On the optimal stopping problem for one-dimensional diffusions. Stochastic Process. Appl., 107 (2):173-212, 2003.

[12] R. A. Doney. Some excursion calculations for spectrally one-sided Lévy processes. In Séminaire de Probabilités XXXVIII, volume 1857 of Lecture Notes in Math., pages 5-15. Springer, Berlin, 2005.

[13] E. Dynkin. Markov processes, Volume II. Springer Verlag, Berlin, 1965.

[14] M. Egami and T. Oryu. An excursion theoretic approach to regulator's bank reorganization problem. Operations Research, to appear in 2015.

[15] M. Egami and K. Yamazaki. Phase-type fitting of scale functions for spectrally negative Lévy processes. Journal of Computational and Applied Mathematics, 264: 1-22, 2014.

[16] M. Egami and K. Yamazaki. Precautional measures for credit risk management in jump models. Stochastics, 85:111-143, 2013.

[17] X. Guo and M. Zervos. $\pi$ options. Stock. Proc. Appl., 120(7):1033-1059, 2010.

[18] A. E. Kyprianou. Introductory lectures on fluctuations of Lévy processes with applications. Universitext. SpringerVerlag, Berlin, 2006.

[19] A. E. Kyprianou and Z. Palmowski. Distributional study of de Finetti's dividend problem for a general Lévy insurance risk process. J. Appl. Probab., 44(2):428-448, 2007.

[20] A. E. Kyprianou and B. A. Surya. Principles of smooth and continuous fit in the determination of endogenous bankruptcy levels. Finance Stoch., 11(1):131-152, 2007.

[21] R. L. Loeffen. On optimality of the barrier strategy in de Finetti's dividend problem for spectrally negative Lévy processes. Ann. Appl. Probab., 18(5):1669-1680, 2008.

[22] B. Øksendal and A. Sulem. Applied Stochastic Control of Jump Diffusions. Springer, New York, 2005.

[23] C. Ott Optimal stopping problems for the maximum process with upper and lower caps Ann. Appl. Probab., 23(6): 2327-2356, 2013.

[24] H. Pham. Continuous-time Stochastic Control and Optimization with Financial Applications, volume 61 of Stochastic Modeling and Applied Probability. Springer, Berlin Heidelberg, 2009.

[25] M. R. Pistorius. On exit and ergodicity of the spectrally one-sided Lévy process reflected at its infimum. J. Theoretical Probab., 17(1):183-220, 2004. 
[26] M. R. Pistorius. An excursion-theoretical approach to some boundary crossing problems and the Skorokhod embedding for reflected Lévy processes. In Séminaire de Probabilités XL, volume 1899 of Lecture Notes in Math., pages 278-307. Springer, Berlin, 2007.

[27] L. Shepp and A. N. Shiraev The Russian option: Reduced regret. Ann. Appl. Probab., 3(3): 631-640, 1993.

[28] B. A. Surya. Evaluating scale functions of spectrally negative Lévy processes. J. Appl. Probab., 45(1):135-149, 2008.

(M. Egami) Graduate School of Economics, Kyoto University, Sakyo-Ku, Kyoto, 606-8501, Japan

E-mail address: egami@econ.kyoto-u.ac.jp

URL: http://www.econ.kyoto-u.ac.jp/ egami/

(T. Oryu) Graduate School of Economics, Kyoto University, SAKyo-Ku, Kyoto, 606-8501, Japan

E-mail address: oryu.tadao.27rest.kyoto-u.ac.jp 